\title{
Definitions of segmental instability in the degenerative lumbar spine and validation of these definitions: a systematic review protocol article according to PRISMA-P 2015 statement
}

\section{Signe Forbech Elmose ( $\square$ signeelmose@hotmail.com )}

Sygehus Lillebalt Middelfart Sygehus https://orcid.org/0000-0003-1522-072X

\section{Gustav Østerheden Andersen}

Sygehus Lillebalt Middelfart Sygehus

\section{Leah Yacat Carreon}

Sygehus Lillebalt Middelfart Sygehus

\section{Freyr Gauti Sigmundsson}

Örebro University Hospital

Mikkel Østerheden Andersen

Sygehus Lillebalt Middelfart Sygehus

\section{Protocol}

Keywords: Systematic review, segmental instability, lumbar, degenerative spondylolisthesis, spinal stenosis, diagnostic imaging, spine surgery

Posted Date: May 17th, 2020

DOI: https://doi.org/10.21203/rs.3.rs-28636/v1

License: (c) (i) This work is licensed under a Creative Commons Attribution 4.0 International License.

Read Full License 


\section{Abstract}

Background The concept of segmental lumbar spine instability is controversial with varying definitions. However, suspicion of instability is used as an indication for vertebral fusion surgery in patients with lumbar degenerative spondylolisthesis and spinal stenosis. The objectives of this systematic review are to describe, the definitions of segmental instability in the degenerative lumbar spine, in studies of patients with lumbar spinal stenosis and/or lumbar degenerative spondylolisthesis, involving diagnostic imaging. And to describe which of the measurable radiological variables used to define instability have been validated against dynamic flexion-extension radiographs.

Method Systematic review conducted according to the Preferred Reporting Items for Systematic Reviews and Meta-Analysis (PRISMA) guidelines. Systematic literature search will be conducted in MEDLINE and EMBASE databases, supplemented by search in Cochrane Library. International Clinical Trials Registry Platform Search Portal and ClinicalTrials.gov will be searched for ongoing or recently completed trials. PROSPERO (International Prospective Register of Systematic Reviews) will be searched before initiating this review. In addition, a gray literature search will be conducted. Studies eligible for inclusion are clinical and biomechanical studies on adult patients with degenerative spondylolisthesis and spinal stenosis and surgical treatment hereof, studies involving diagnostic imaging. We will include studies giving a definition or describing patho-anatomical findings of segmental instability or any synonym or antonym hereof. Two reviewers will independently screen articles, involving a third reviewer as referee. Screening process and data extraction conducted using Covidence software. Data synthesis presented in results checklist and systematic narrative synthesis. Data synthesis on secondary objective by contingency table.

Discussion With this systematic review we want to contribute to evidence based treatment planning of lumbar degenerative spondylolisthesis with spinal stenosis. We also aim to present our result in a comprehensive manner, to encourage consensus regarding the definition of segmental instability. Registration This protocol was submitted to the International Prospective Register of Systematic Reviews (PROSPERO) on 29. April 2020, submission ID: 182827

\section{Background}

Age related changes in the lumbar spine could be associated with structural alternations affecting the alignment of the vertebrae.

Lumbar degenerative spondylolisthesis (LDS) is forward slippage of the proximal vertebra over a more distal vertebra caused by degeneration and weakening of the surrounding structures. This leads to development of lumbar spinal stenosis (LSS), causing radicular leg pain (neurogenic claudication) with or without back pain. Surgical treatment with decompression of the neural structures have been shown to effectively relieve symptoms and improve health-related quality of life(1). However, there are patients who have a dynamic component to the segmental instability associated with LDS. With radiological signs of LDS and/or instability, the surgeon may choose to perform a fusion(2,3). Fusion surgery is a more 
complex procedure, has a higher cost and higher risk of complications than decompression alone $(4,5)$. Fusion might also lead to adjacent segment disease.

However, a common indication for reoperation, usually a fusion, following decompression is iatrogenic instability. The incidence varies in the literature ranging from $0 \%$ to $63 \%(6)$. Evaluating the risk for postoperative instability is important in the preoperative patient assessment.

However, the diagnosis or concept of segmental lumbar spine instability is controversial. The first study addressing the term dates back to 1944(7). This and other studies investigated segmental motion on lateral flexion and extension radiographs, which is still considered the current gold standard(7-9). However, in spine surgery, magnetic resonance imaging (MRI) is increasingly the preferred diagnostic imaging modality, since it is superior to radiograph in demonstration central, thickening of the ligamentum flavum and facet joint degenerative alterations. Additionally, MRI does not expose the patient to radiation. Several studies have found MRI characteristics associated with clinical and radiological instability $(2,10-20)$. These include facet joint effusion, disc degeneration/height, degenerative spondylolisthesis, facet joint angle and facet joint morphology.

In 2015, Simmonds et al published a review of studies published from 1990 to 2013 with the primary objective to identify measurable preoperative radiographic variables to define spinal instability in patients with degenerative spondylolisthesis(2). According to Simmonds et al, the literature varies in definition of instability in patients with LDS, and the evidence on how to diagnose instability are of low quality(2).

Often studies lack a definition of instability or define instability with reference to studies on normal individuals $(2,6,10)$. In general, the criteria for instability varies amongst studies. Guha et al published a literature review in 2015 presenting 24 studies on degenerative lumbar stenosis to establish the incidence of iatrogenic instability(6). They found that 10 out of 24 studies reported incidences of postoperative instability without specifying its definition, and the remaining defined instability as an increase in sagittal translation by varying length or percentage(6).

Rationale:

Since lumbar segmental instability is used as an indication for fusion in addition to decompression alone, there needs to be validated and evidence-based definition of instability, like all other diagnostic tools or tests. In order to facilitate the process, it is necessary to describe the different definitions of instability and the evidence supporting them.

Treatment optimizing approaches for patients with instability require better knowledge of the accuracy of the diagnostic images that are used in daily clinical practice.

\section{Objectives:}

With this systematic review we want to contribute to evidence based treatment planning of lumbar degenerative spondylolisthesis with spinal stenosis. We also aim to present our result in a comprehensive 
manner, to encourage consensus regarding the definition of segmental instability both in daily clinical setting and in prospective research.

Primary objective

To describe, the definitions of segmental instability in the degenerative lumbar spine, in studies of patients with lumbar spinal stenosis and/or lumbar degenerative spondylolisthesis, involving diagnostic imaging.

Secondary objective

Which of the measurable radiological variables used to define instability have been validated against dynamic flexion-extension radiographs?

\section{Methods}

\section{Study design}

The systematic review conducted according to PRISMA guidelines(21)

This protocol article is conducted following the PRISMA-P guidelines(22, 23). A copy of the PRISMA-P check list provided (see additional file 1)(22-24).

\section{Eligibility_criteria:}

A broad perspective is necessary when developing the search string and eligibility criteria, studies might define instability without it being a part of the objective or outcome.

Types of studies: We will include the following types of studies: 1) clinical studies regardless of design 2) biomechanical studies 3 ) conference abstracts 4) reference programs and guidelines from various national and international associations and societies with relevance to spine surgery.

We will exclude patient handouts, newspaper articles, audio-video media, legal cases, news and interviews. Furthermore, we will exclude commentaries, letters and editorials during screening.

We choose to include Epubs ahead of print. There will be no restriction to year of publication.

In the following, the eligibility criteria will be elaborated using the PICO (participants, intervention, comparators and outcome) elements.

\section{Participants:}

We will include studies on patients with lumbar DS and LSS. We will include adult participants $(>18$ years of age) regardless of history of back pain or radiculopathy to avoid bias. Hereby we will not include "degenerative" or synonyms of the term as a block in the search string. 
We will exclude studies on patients with malignancy or undergoing treatment for malignancy, spinal fracture patients or scoliosis patients with a Cobb angle $>20 \rrbracket$. Furthermore, non-human studies will be excluded.

\section{Intervention:}

We will include studies describing surgical treatment for LDS and LSS involving diagnostic imaging. We will include studies describing or investigating indicators for fusion surgery for LDS and LSS.

Surgical treatment meaning surgical decompression of neural structures of the spine or decompression with fusion of vertebrae. No restrictions in regard to type of decompression or fusion. We will include studies comparing surgical and nonsurgical treatment of instability and LDS and LSS involving diagnostic imaging.

Diagnostic imaging for inclusion MRI, computed tomography (CT) scan, CT-myelograms or flexionextension or lateral radiographs of the lumbosacral spine. We will include studies on both pre and postsurgery imaging.

Studies might not specify using diagnostic imaging in the abstract, but it is implied that diagnosing patients with LDS and LSS requires diagnostic imaging. These studies will be included for full text screening.

We will include biomechanical studies on segmental instability involving diagnostic imaging.

Studies describing or investigating non-surgical treatment for LDS and LSS will be excluded, regardless of diagnostic imaging.

\section{Comparators:}

We will include studies comparing indicators of instability between different diagnostic imaging modalities mentioned above. Including validation and association studies. We will include both comparative and non-comparative studies.

\section{Outcome:}

Since not all studies will state instability as part of the study purpose or as an outcome in the abstract or title. Studies may, however, still provide a definition or description in the full text. The definition can be narrative, qualitative or quantitative.

Of interest are studies that investigate patho-anatomical findings and characteristics of instability on different diagnostic imaging modalities.

We will include studies regardless of stating instability as an outcome. 
Instability has several synonyms and several ways to describe the condition can be found in the literature. To avoid bias due to terminology the following terms will be accepted: dynamic spondylolisthesis, slippage, slip, translation, subluxation, segmental or segment motion and abnormal movement.

Also, of interest are studies investigating the antonym "stability" as a general term.

Studies reporting one or more of the outcomes listed below or surrogates thereof will be included.

Interpretation of MRI, CT-scans and/or radiographs of the lumbar spine, describing:

- Intervertebral anterior-posterior or lateral translation/slip/slippage/movement and/or rotation.

- Intervertebral disc height and angle

- Intervertebral disc degeneration

- Degenerative changes in the vertebra, facet joints or intervertebral discs

- Facet joint morphology

We exclude studies if no definition of instability, or synonym thereof, is given in the full text.

\section{Timing.}

There will be no restriction based on the length or lack of follow-up.

\section{Setting.}

There will be no restriction by type of setting.

\section{Language:}

We will include English, Danish, Norwegian, Swedish.

\section{Information sources and search strategy.}

Literature search strategies will be developed by block building, using medical subject headings (MeSH) and exploded when necessary. Floating subheadings used appropriately. Free text and keyword search with relevant synonyms and antonyms. Search strategy will be developed with assistance of research librarian affiliated with University Library of Southern Denmark, University of Southern Denmark.

We will search MEDLINE (Ovid SP interface, 1948 and onwards), EMBASE (Ovid SP interface, 1947 and onwards), Cochrane Library (Wiley interface). As well, International Clinical Trials Registry Platform Search Portal (ICTRP, World Health Organization) and ClinicalTrials.gov (U.S. National Library of Medicine) will be searched for ongoing or recently completed trials. PROSPERO (International Prospective Register of Systematic Reviews, National Institute of Health Research) will be searched for ongoing or 
recently completed systematic reviews. In addition, a gray literature search of relevant national and international societies of spine surgery and congress or conference proceedings will be conducted.

See Additional file 2 for proposed strategy to be run in MEDLINE. After adaptation similar structured search strategies will be designed using syntax and subject headings appropriate for each database listed above.

Searches to be end conducted May 2020.

No language restriction will be applied to the electronic searches. We will not use any methodological search filters since this could lower sensitivity.

\section{Searching other resources}

Reference list of all primary studies identified by our search.

\section{Literature selection}

Titles and abstracts of all records identified by the search will be initially screened for potential inclusion by two independent researchers (SFE and GØA). If the study type is unclear from abstract, or no abstract is available, but the title suggests a potentially relevant study, the full text will be reviewed. A full text of all the potentially relevant articles will be obtained and a full text screening for final inclusion conducted by same independent researchers (SFE and GØA). Any disagreement will be resolved by discussion and by a tertiary reviewer (MA), functioning as a referee. To ensure consistency across reviewers, we will conduct calibration exercises before starting the screening process. Reasons for exclusion will be noted. If necessary, additional information will be sought form study authors to resolve questions about eligibility. References are exported to EndNote X9(25) and exported to COVIDENCE software(26). Screening and selection will be carried out using COVIDENCE. Any duplicates will be excluded. The process of screening and selection will be documented and reported using PRISMA flowchart(21).

Summary on selected studies included to be presented in Microsoft Word Table (Microsoft Office, Redmond, WA) with the following information: study characteristics as author, year of publication, journal name, study design, study size and participants characteristics, diagnosis, type of surgery and assessment pre or post-surgery and outcomes.

\section{Data extraction and management}

The data will be extracted by one reviewer (SFE) with verification by second reviewer (MA) to reduce bias and risk of errors. Before commencement calibration exercises will be performed. A pre-formatted Microsoft Excel (Microsoft Office, Redmond, WA) spreadsheet will be used to extract data form the studies.

Data presented in a Microsoft Word Table (Microsoft Office, Redmond, WA) result checklist customized for this review (see additional file 3). Any disagreement to be resolved by discussion with a third reviewer 
as referee (FGS) by unresolved disagreements. By uncertainties, the study author will be contacted by email, maximum of three attempts.

\section{Outcomes and prioritization}

The primary outcome is definition of segmental instability. It can be presented as a numeric measurement or narrative. For the definition of instability, any reference to other studies' or author's definition of instability is included and if the conclusion states a new definition of instability. If data is presented graphically and no numerical data is presented, the authors will be contacted using the contact information listed in the article. A maximum of three email attempts will be made to obtain missing information.

Secondary outcomes are validation of diagnostic measurement used to define instability: inter-examiner reliability, intra-examiner reliability, intra and inter-reproducibility.

The results checklist table will present extracted data. Measurements will be reported as stated either as absolute numbers or as relative numbers. If possible, the absolute numbers will be calculated.

Variables in checklist and definition:

- Study identification from summary table

- Definition: the definition of segmental instability stated in the study

- Diagnostic imaging: naming the radiological modality used/investigated.

- Characteristics: stating the characteristics of the radiological technology.

- Patient position: during diagnostic imaging, e.g. supine, prone or standing

- Spinal level: number/numbers of vertebrae

- Measurement outcomes: which vertebral measurements are stated to be relevant to instability

- Method of measuring: keywords describing the method to measure outcomes

- Validation data: inter-examiner reliability, intra-examiner reliability, intra and inter-reproducibility

Assessment of methodological quality.

Since data extracted for the primary objective of this review are not related to the study design no study quality assessment necessary.

However, it is relevant to consider risk of bias in relation to the secondary objective. One reviewer will independently asses risk of bias of the included studies relevant for secondary objective. Verification by second reviewer. Risk of bias to asses internal validity of each study on outcome level for either observational studies or randomized controlled trials accordingly. Judgement options will be high, low or unclear risk of bias.

Calibration exercises to be conducted before assessment start. 


\section{Data synthesis}

Data answering primary objective are not expected to be suitable for a quantitative synthesis or metaanalysis. However, it will be presented in the results checklist. Furthermore, to clarify and illustrate the result a flow diagram will be constructed. The flow diagram will present the interlinked definitions and the cross references (with or without modifications). This would also be user friendly to the spine surgeon for whom this review is intended. Furthermore, a systematic narrative synthesis will be provided with information supporting the table and flow diagram and explaining the characteristics and findings of the included studies.

In regard to the secondary objective data from the result checklist will be isolated and if possible, presented quantitatively in a contingency table, with flexion - and extension radiographs as gold standard compared to different radiographic measurements.

\section{Discussion}

The concept of segmental lumbar spine instability is controversial. The literature varies in definition of instability in patients with LDS, and a definition is not always reported $(2,6)$. With this systematic review, we aim to describe the different definitions of instability in the literature and the evidence supporting them. However, this systematic review will have its limitations.

A definition of instability or stability might be given within the full text but not in the abstract or title. Thereby the study would not necessarily be indexed in the database as being of relevance. It is necessary to have a broad perspective when developing the search strategy to avoid introducing bias. We are aware that this strategy will give a low search specificity. However, a two-step screening process by independent researchers will help increase the specificity of the overall review.

Previous review by Simmonds et al(2) chose to limit the time period, but this might eliminate early important studies. As mentioned, the first study on instability dates back to 1944 , and we aim to get a full historical perspective of the concept segmental instability.

An assessment of the risk of bias is an important part of any systematic review(22). However, our primary objective is not influenced by study design, and a critical appraisal of the methodology quality are not relevant. However, our secondary objective, investigation of validity, can be influenced by bias. Therefore, we chose to incorporate an assessment of risk of bias, when addressing this objective.

It could seem relevant to address reporting bias, since we anticipate that not all studies will give a definition of instability. However, we have chosen to focus on describing the existing definitions. An investigation of the frequency of reporting a definition of instability could be an objective for a future review.

We are confident that by constructing a search string in cooperation with a research librarian and by writing a thorough protocol, we have constructed a solid base for a systematic review that will encourage 
consensus regarding the definition of segmental instability both in daily clinical setting and in prospective research.

\section{Abbreviations}

PRISMA-P: Preferred Reporting Items for Systematic Reviews and Meta-Analysis protocols; PRISMA: Preferred Reporting Items for Systematic Reviews and Meta-Analysis; PROSPERO: the International Prospective Register of Systematic Reviews; LDS: lumbar degenerative spondylolisthesis; LSS: lumbar spinal stenosis; MeSH: medical subject headings; ICTRP: International Clinical Trials Registry Platform Search Portal

\section{Declarations}

Ethics approval and consent to participate

Not applicable

Consent for publication

Not applicable

Availability of data and material

Not applicable

Competing interests

The authors declare that they have no competing interests.

\section{Funding}

no funding or financial support

Authors contributions

o Protocol manuscript: SFE with assistance from MA, FGS, LC

o Literature search: SFE with assistance from research librarian

o Article selection/study screening: SFE and GØA, referee MA

o Data extraction, assessment of bias: SFE verification by MA, referee FGS

$\S$ Contact to study authors if relevant: SFE

o Systematic review manuscript: SFE 
$\S$ Edited and reviewed by FGS, MA and LC

\section{Acknowledgements}

The authors acknowledge the great help form research librarian Mette Brandt Eriksen, Ph.D., University Library of Southern Denmark, University of Southern Denmark (SDU), for her contribution and support in developing the search strategy.

\section{References}

1. Paulsen RT, Bouknaitir JB, Fruensgaard S, Carreron L, Andersen M. Patient are satisfied one year after decompression surgery for lumbar spinal stenosis. 2016;1(11).

2. Simmonds AM, Rampersaud YR, Dvorak MF, Dea N, Melnyk AD, Fisher CG. Defining the inherent stability of degenerative spondylolisthesis: a systematic review. J Neurosurg Spine. 2015;23(2):178-89.

3. Ghogawala Z, Dziura J, Butler WE, Dai F, Terrin N, Magge SN, et al. Laminectomy plus Fusion versus Laminectomy Alone for Lumbar Spondylolisthesis. N Engl J Med. 2016;374(15):1424-34.

4. Ulrich NH, Burgstaller JM, Pichierri G, Wertli MM, Farshad M, Porchet F, et al. Decompression Surgery Alone Versus Decompression Plus Fusion in Symptomatic Lumbar Spinal Stenosis: A Swiss Prospective Multicenter Cohort Study With 3 Years of Follow-up. Spine (Phila Pa 1976). 2017;42(18):E1077-E86.

5. Forsth P, Olafsson G, Carlsson T, Frost A, Borgstrom F, Fritzell P, et al. A Randomized, Controlled Trial of Fusion Surgery for Lumbar Spinal Stenosis. 2016;1(15):1413-23.

6. Guha D, Heary RF, Shamji MF. latrogenic spondylolisthesis following laminectomy for degenerative lumbar stenosis: systematic review and current concepts. Neurosurg Focus. 2015;39(4):E9.

7. Knutsson F. The Instability Associated with Disk Degeneration in the Lumbar Spine. Acta Radiologica. 1944;25(5-6):593-609.

8. AHO A, TAHTI E. Significance of functional radiography of the lumbar spine in forward and backward flexion. Ann Chir Gynaecol Fenn. 1957;46(3):336-50.

9. Boden SD, Wiesel SW. Lumbosacral segmental motion in normal individuals. Have we been measuring instability properly? Spine (Phila Pa 1976). 1990;15(6):571-6.

10. Boden SD, Davis DO, Dina TS, Patronas NJ, Wiesel SW. Abnormal magnetic-resonance scans of the lumbar spine in asymptomatic subjects. A prospective investigation. J Bone Joint Surg Am. $1990 ; 72(3): 403-8$.

11. Snoddy MC, Sielatycki JA, Sivaganesan A, Engstrom SM, McGirt MJ, Devin CJ. Can facet joint fluid on MRI and dynamic instability be a predictor of improvement in back pain following lumbar fusion for 
degenerative spondylolisthesis? Eur Spine J. 2016;25(8):2408-15.

12. Ben-Galim P, Reitman CA. The distended facet sign: an indicator of position-dependent spinal stenosis and degenerative spondylolisthesis. Spine J. 2007;7(2):245-8.

13. Hasegawa K, Kitahara K, Shimoda H, Hara T. Facet joint opening in lumbar degenerative diseases indicating segmental instability. J Neurosurg Spine. 2010;12(6):687-93.

14. Lattig F, Fekete TF, Kleinstuck FS, Porchet F, Jeszenszky D, Mannion AF. Lumbar facet joint effusion on MRI as a sign of unstable degenerative spondylolisthesis: should it influence the treatment decision? J Spinal Disord Tech. 2015;28(3):95-100.

15. Even JL, Chen AF, Lee JY. Imaging characteristics of "dynamic" versus "static" spondylolisthesis: analysis using magnetic resonance imaging and flexion/extension films. Spine J. 2014;14(9):1965-9.

16. Kuhns BD, Kouk S, Buchanan C, Lubelski D, Alvin MD, Benzel EC, et al. Sensitivity of magnetic resonance imaging in the diagnosis of mobile and nonmobile L4-L5 degenerative spondylolisthesis. Spine J. 2015;15(9):1956-62.

17. Chaput C, Padon D, Rush J, Lenehan E, Rahm M. The significance of increased fluid signal on magnetic resonance imaging in lumbar facets in relationship to degenerative spondylolisthesis. Spine (Phila Pa 1976). 2007;32(17):1883-7.

18. D'Anastasi M, Birkenmaier C, Schmidt GP, Wegener B, Reiser MF, Baur-Melnyk A. Correlation between vacuum phenomenon on CT and fluid on MRI in degenerative disks. AJR Am J Roentgenol. 2011;197(5):1182-9.

19. Rihn JA, Lee JY, Khan M, Ulibarri JA, Tannoury C, Donaldson WF, 3rd, et al. Does lumbar facet fluid detected on magnetic resonance imaging correlate with radiographic instability in patients with degenerative lumbar disease? Spine (Phila Pa 1976). 2007;32(14):1555-60.

20. Schleich C, Muller-Lutz A, Blum K, Boos J, Bittersohl B, Schmitt B, et al. Facet tropism and facet joint orientation: risk factors for the development of early biochemical alterations of lumbar intervertebral discs. Osteoarthritis Cartilage. 2016;24(10):1761-8.

21. Moher D, Liberati A, Tetzlaff J, Altman DG, Group P. Preferred reporting items for systematic reviews and meta-analyses: the PRISMA statement. J Clin Epidemiol. 2009;62(10):1006-12.

22. Moher D, Shamseer L, Clarke M, Ghersi D, Liberati A, Petticrew M, et al. Preferred reporting items for systematic review and meta-analysis protocols (PRISMA-P) 2015 statement. Syst Rev. 2015;4:1.

23. Shamseer L, Moher D, Clarke M, Ghersi D, Liberati A, Petticrew M, et al. Preferred reporting items for systematic review and meta-analysis protocols (PRISMA-P) 2015: elaboration and explanation. BMJ. 2015;350:g7647. 
24. Moher D, Stewart L, Shekelle P. Implementing PRISMA-P: recommendations for prospective authors. Syst Rev. 2016;5:15.

25. Team TE. EndNote. EndNote X9 ed. Philadelphia, PA: Clarivate Analytics; 2013. p. 64 bit.

26. COVIDENCE. Melbourne, Australia: Veritas Health Innovation; 2019. p. Covidence systematic review software.

\section{Supplementary Files}

This is a list of supplementary files associated with this preprint. Click to download.

- Additionalfile3.docx

- Additionalfile2.docx

- Additionalfile1.docx 